\title{
Molecular Diagnostics in Breast Cancer Routine Practice
}

Natalie D ter Hoeve, Cathy B Moelans, Willemijne AME Schrijver, Wendy de Leng and Paul J van Diest

Department of Pathology, University Medical Center Utrecht, The Netherlands

DOI: https://doi.org/10.17925/EOH.2017.13.01.74

$\mathrm{T}$

he portfolio of adjuvant systemic treatment of breast cancer nowadays contains novel anti-hormonal and chemotherapeutic drugs, immunotherapeutic approaches and small molecules that are only effective in a limited number of patients and are often associated with high costs and significant side effects. Therefore, a personalised approach based on individual tumour biomarkers is required to arrive at the optimal balance between effectiveness on the one hand, and costs and side effects on the other. The aim of this paper is to provide an overview of the molecular biomarkers and associated molecular tests that are currently relevant in pathology of invasive breast cancer.

\section{Keywords}

Breast cancer, pathology, molecular biomarkers

Disclosure: Natalie D ter Hoeve, Cathy B Moelans, Willemijne AME Schrijver, Wendy de Leng and Paul J van Diest have nothing to disclose in relation to this article. This study involves a review of the literature and did not involve any studies with human or animal subjects performed by any of the authors. No funding was received for the publication of this article.

Authorship: All named authors meet the International Committee of Medical Journal Editors (ICMJE) criteria for authorship of this manuscript, take responsibility for the integrity of the work as a whole, and have given final approval to the version to be published.

open Access: This article is published under the Creative Commons Attribution Noncommercial License, which permits any non-commercial use, distribution, adaptation and reproduction provided the original author(s) and source are given appropriate credit.

Received: 12 October 2016

Accepted: 13 February 2017

Citation: European Oncology \& Haematology, 2017;13(1):74-9

Corresponding Author: Paul J van Diest, Department of Pathology, University Medical Center Utrecht, PO Box 85500, 3508 GA Utrecht. E: p.j.vandiest@umcutrecht.nl
Adjuvant systemic treatment of breast cancer is moving away from the limited portfolio of traditional hormonal drugs and chemotherapy, towards a gamma of novel anti-hormonal and chemotherapeutic drugs, immunotherapeutic approaches and small molecules. All these therapeutic approaches are unfortunately effective in a limited number of patients and are often associated with high costs and significant side effects. Therefore, the traditional "one size fits all" approach can no longer be upheld, and a personalised approach based on individual tumour biomarkers is required to arrive at the optimal balance between effectiveness on the one hand and costs and side effects on the other.

Over recent years, progress in molecular techniques has made it possible to analyse formalin fixed paraffin embedded (FFPE) tumour material of larger cohorts of patients. This has incentivised many translational studies relating molecular tumour biomarkers to diagnosis, prognosis and/or response to therapy, yielding many relevant molecular biomarkers that have rather quickly made it to clinical pathology practice. The aim of this paper is to provide an overview of the molecular biomarkers and associated molecular tests that are currently relevant in pathology of invasive breast cancer.

\section{Diagnostic markers Hereditary breast cancers}

About $5-10 \%$ of breast cancer cases are due to a hereditary predisposition. ${ }^{1}$ In most of these cases, mutations are found in well-characterised, medium to high-risk genes, such as BRCA1, BRCA2, CHEK2, TP53, PALB2, or BRIP1, ${ }^{2}$ of which BRCA1 and BRCA2 are the most important ones. Promotor hypermethylation of $B R C A 1$ and $B R C A 2$ seems to be very infrequent in $B R C A 1 / 2$ germline mutation related breast cancers, and significantly more frequent in sporadic cancers [data unpublished, submitted for publication]. BRCA1/2 promoter methylation testing, pointing to sporadic cancers when present, may grow out to be clinically useful once the diagnostically optimal CPG islands have been identified. ${ }^{3}$

Copy number analysis by array comparative genomic hybridisation (CGH) showed frequently occurring gains of $3 q, 7 p, 8 q 10 p, 12 p, 16 p$ and 17q, and loss of 2q, 3p, 4p, 4q, 5q, 12q, 16p and $18 \mathrm{q}$ in BRCA1 germline mutation related cancers. BRCA2 related breast cancers show more frequently gains of $8 q, 17 q 22-q 24$ and 20q13, and loss of $8 p, 6 q, 11 q$ and $13 q$ compared to BRCA1 related cancers. ${ }^{4,5}$ A multiplex ligation-dependent probe amplification (MLPA) kit $^{\circledR}$ (MRC Holland, Amsterdam, The Netherlands) for copy number testing pointing to BRCA1 related cancers is commercially available.

\section{Molecular (intrinsic) typing}

Several gene expression studies have revealed the existence of five molecular subtypes of breast cancer: a "basal-like" subgroup with low oestrogen receptor (ER)/progesterone receptor(PR)/ human epidermal growth factor receptor 2 (HER2) and expression of basal cytokeratins; a subgroup mainly driven by HER2 amplification and overexpression while being ER/PR Iow; 
a luminal A group with high ER/PR and low HER2; and a luminal B group that is also ER/PR high but with additional HER2 overexpression and/ or high proliferation. ${ }^{6,7} \mathrm{~A}$ further "normal breast like" group is likely the result of absence of tumour in the frozen pieces that were analysed without morphological control by a pathologist. These different classes have varying clinical behaviour and have led to a new way of thinking about classification of breast cancer going beyond morphology. Nevertheless, good correlations exist between these intrinsic subtypes and morphology. The basal-like group contains high grade ductal medullary and metaplastic cancers as well as the low grade salivary gland type cancers (adenoid cystic cancers [AdCC], acinic cell cancers, myoepithelial cancers). The HER2 group contains poorly differentiated HER2 overexpressing ductal and apocrine cancers. The luminal A group contains mainly low grade ductal, lobular, ductulolobular, tubular, cribriform, mucinous and micropapillary cancers. Several intrinsic typing tests are commercially available (PAM50 ${ }^{\circledR}$, Nanostring, Washington, US; Blueprint ${ }^{\circledast}$, Agendia, Amsterdam, The Netherlands). However, these intrinsic subtypes have little added value to type, grade and expression of ER/PR/HER2, and the reproducibility of intrinsic subtyping based on gene expression has proven to be low across datasets and technology platforms. Besides, these tests are not available for local testing in pathology labs, are time consuming and expensive. Therefore, a more practical approach is probably to use an immunohistochemical surrogate as depicted in Table 1.

PAM50 and Blueprint are two commercially available gene expression test for intrinsic subtyping (from centrally at Nanostring and Agendia, respectively). The PAM50 signature employs 50 genes and can be applied on FFPE material. ${ }^{8}$ Blueprint contains 80 genes and can be applied on FFPE material as well.

\section{Translocations \\ Adenoid cystic carcinoma}

While being a frequent cancer type in the minor and major salivary glands with frequent perineural invasion and poor prognosis, AdCC is a rare cancer type in the breast, accounting for $0.1-1 \%$ of breast cancers, with infrequent perineural invasion and indolent clinical behaviour despite their triple negative (ER/PR/HER2) state. ${ }^{9}$ These carcinomas often display the recurrent chromosomal translocation $\mathrm{t}(6 ; 9)$ (q22e23;p23e24), which generates oncogenic fusion transcripts involving the two transcription factor genes MYB and NFIB. In the $t(6 ; 9)$ (q22eq23;p23ep24), the exon 14 of MYB is fused to the final coding exons of $N F I B$, usually due to breakpoints in MYB intron 14 and intron 8 in NFIB. The fusion results in loss of the $3^{\prime}$-end of $M Y B$, including several conserved binding sites for microRNAs that regulate $M Y B$ expression negatively. More recently, also recurring $t(8 ; 9)$ and $t(8 ; 14)$ translocations have been described fusing the MYBL1 gene to the NFIB and RAD51B genes, respectively. ${ }^{10,11}$ Due to the characteristic histological features of AdCC, translocation assays may not be often necessary in diagnostic practice, but may be assessed by fluorescent in situ hybridisation (FISH) kits or reverse transcription polymerase chain reaction (RT-PCR) in difficult cases.

\section{Secretory cancer}

Secretory cancer is a rare breast cancer type that occurs at all ages, even at quite young age. Recently, it was shown that secretory cancer is characterised by a balanced chromosomal translocation $t(12 ; 15)$ (p13;q25), which leads to the formation of an oncogenic ETV6-NTRK3 fusion gene encoding a chimeric tyrosine kinase, also demonstrated in paediatric mesenchymal cancers. ${ }^{12}$ Translocations may be assessed by FISH or RT-PCR in difficult cases.
Table 1: Immunohistochemical surrogate for intrinsic subtyping based on expression of ER/PR/HER2 and Ki67, basal cytokeratins and EGFR

\begin{tabular}{|l|l|l|l|l|l|l|}
\hline & ER & PR & HER2 & Ki67 & $\begin{array}{l}\text { Basal cytokeratins } \\
\text { (CK5/6, CK14) or EGFR }\end{array}$ \\
\hline Luminal A & + & + or - & - & & \\
\hline Luminal B & + & + or - & overexpressed & & \\
\hline & + & + or - & & high & \\
\hline HER2 driven & - & - & overexpressed & & \\
\hline Basal-like & - & - & - & & + \\
\hline
\end{tabular}

$E G F R=$ epidermal growth factor receptor; $E R=$ oestrogen receptor; $H E R 2$ = human epidermal growth factor receptor 2; $P R=$ progesterone receptor.

\section{Prognostic markers \\ Gene expression tests}

Several gene expression tests have been developed to predict prognosis and response to therapy. The above described PAM50 has proven prognostic value. ${ }^{8}$ Further popular prognostic gene expression tests are EndoPredict ${ }^{\circledR}$ (Sividon Diagnostics, Köln, Germany), MammaPrint ${ }^{\circledR}$ (Agendia, Amsterdam, The Netherlands) and Oncotype Dx ${ }^{\circledR}$ (Genomic Health, California, US).

EndoPredict is an 8-gene RT-PCR test that primarily targets ER+/HER2patients that have 0-3 lymph node metastases. It can be applied on FFPE and has been validated for local application in pathology labs, and is available from Sividon Diagnostics. It was proven to be prognostically important in several studies (level I-II evidence), especially in combination with lymph node status and tumour size, ${ }^{13}$ and gives a binary prognostic indication. Mammaprint is a 70-gene microarray expression test that primarily targets stage I-II breast cancer patients with tumour size $<5 \mathrm{~cm}$. It was originally developed as a prognostic factor with evidence level I on frozen material but was recently also validated on FFPE material. ${ }^{14}$ It is available only centrally from Agendia and gives a binary prognostic indication. Oncotype Dx is a 21 gene RT-PCR test (including five reference genes) targeting ER+ patients with no lymph node metastases. It is available only centrally from Genomic Health and gives a three-tiered prognostic indication. ${ }^{15}$

These gene expression tests in general provide good prognostic information and are analytically well validated except. A recent paper indicated that these tests may be significantly suffer from intra-tumour heterogeneity. ${ }^{16}$ They are recommended in most guidelines like AGO, St Gallen, The European Society for Medical Oncology (ESMO), The American Society of Clinical Oncology (ASCO), National Comprehensive Cancer Network (NCCN), and The National Institute for Health and Care Excellence (NICE). A hurdle in broader application is the high costs at about $€ 3000$ per test. The added prognostic value to type and grade is likely limited, ${ }^{17}$ but their better reproducibility are arguments in favour. No prognostic comparative studies have been performed, so decisions on the preferred test are usually taken based on local arguments.

\section{IHC4}

Although the immunohistochemistry $(\mathrm{IHC}) 4$ score is based on $\mathrm{IHC}$ based protein expression of ER $\alpha, P R, H E R 2$ and Ki67 and thereby not strictly a molecular test, it is discussed here since it has been proposed as a cheap alternative to gene expression tests. IHC4 provided similar prognostic information as Oncotype Dx score in a study of ER-positive patients from the ATAC (Arimidex, Tamoxifen, Alone or in Combination) trial who did not receive adjuvant chemotherapy..$^{18}$ In addition, PAM50 and $\mathrm{IHC} 4$ scores provided comparable prognostic information in the 
HER2-negative/node-negative group. ${ }^{19}$ Quantitative evaluation of ER, PR and HER2 status is well standardised and guidelines for Ki67 assessment are available. ${ }^{20} \mathrm{IHC} 4$ could significantly improve the routine availability of prognostic information in a cost-effective manner.

\section{Predictive markers}

\section{Gene expression tests}

The value of the above described gene expression tests EndoPredict, MammaPrint and Oncotype Dx lies in their predictive value rather than their prognostic power in view of their high costs. If patients that are deemed eligible for chemotherapy based on classic prognostic factors can be safely spared chemotherapy when their gene expression test turns out to be favourable, much money can be spared elsewhere and application may be cost-effective. All these tests have proven to have such predictive value at level I evidence, although lack of intra-tumour heterogeneity studies remains a weak point. Further, comparative predictive studies have been performed, so decisions on the preferred gene expression test are usually taken based on local preferences rather than firm scientific data.

\section{ER $\alpha$ mRNA expression}

Since $1 \mathrm{HC}$ has its limitations, ESR1 mRNA expression has been explored as an alternative. There appeared to be a very good correlation between ESR1 mRNA expression by microarray analysis and ER $\alpha \mathrm{IHC}{ }^{21}$ There is no proven predictive superiority of gene expression in mRNA-protein discrepant cases. The "Targetprint" test (Agendia, Amsterdam, The Netherlands) was recently taken off the market.

\section{ESR1 mutations}

ER $\alpha$ expression has for a long time served as indication for hormonal therapy. More recently, molecular changes in ESR1, the gene on chromosome 6 encoding $E R \alpha$, have been demonstrated to relate to endocrine responsiveness as well. ESR1 mutations leads to conformation change which mimics activated ligand-bound receptor and induces ligand-independent ER activity,22 resulting in tumour growth despite endocrine therapy. The mutants appear to retain some sensitivity to drugs directly targeting the receptor suggesting potential pharmacologic strategies for these patients..$^{23}$

Although ESR1 mutations are very uncommon in primary breast tumours, ${ }^{24}$ metastases more frequently harbour mutations in the ligandbinding domain resulting in endocrine resistance. ${ }^{25,26}$ Interestingly, these mutations have been demonstrated in blood providing a monitoring option. ${ }^{27}$ Breast tumours of women naïve for endocrine therapy do not seem to harbour these mutations, suggesting either the clonal selection of very rare resistant primary tumour clones or their later acquisition under the pressure of endocrine treatments. ${ }^{25}$ Relatively many patients develop polyclonal mutations. Aromatase inhibitor treatment in the metastatic setting rather than in the adjuvant setting induces ESR1 mutations. ${ }^{28}$ The pan-phosphatidylinositol 3-kinase (PI3K) inhibitor pictilisib and the CDK4/6-inhibitor palbociclib do not seem to be effective in ESR1 mutated patients, ${ }^{29}$ and ESR1 mutations did not indicate impaired survival on fulvestrant compared to ESR1 wildtype. On examestane, however, ESR1 mutations do seem to indicate worse prognosis. Overall, ESR1 mutations seem to be associated with more aggressive disease biology.29

\section{ESR1 amplification}

The frequency of ESR1 amplification is highly debated. ESR1 gains and amplifications were originally described in $15 \%$ and $20 \%$ of breast cancer samples, respectively. ${ }^{30}$ In these studies, ESR1 copy number increase was correlated to high ER $\alpha$ protein expression, and was associated with very good response to tamoxifen and good survival. In later studies, the ESR1amplification frequency was 0-23\% when FISH was used for detection, and less than 5\% when biochemical assays, CGH, MLPA, and GPCR, were used. ${ }^{31}$ In general, the amplification frequency is highly concordant between the different studies that used biochemical assays, whereas considerable variability exists among the studies that used FISH (different protocols, scoring systems, artefacts). Most amplifications are low level, and FISH may be hampered by presence of pre-mRNA. ${ }^{31}$

\section{ESR1 promoter hypermethylation}

ESR1 promoter methylation has been suggested as an alternative mechanism of ER $\alpha$ expression loss. Methylation of the ESR1 promoter is found in a proportion of the ER negative tumours. ${ }^{32}$ and in ER negative cells lines. The use of demethylating agents was shown to reactivate ER expression which reveals a potential new strategy to overcome endocrine resistance in breast cancer patients. ${ }^{33}$ ESR1 promoter methylation analysis has however not yet reached the status of a clinical test.

\section{HER2 amplification}

The HER2 is a proto-oncogene amplified in 10-15\% of human breast cancers. HER-2/neu (HER2) status predicts prognosis and sensitivity to HER2 protein targeting drugs such as trastuzumab, pertuzumab and lapatinib. Accurate assessment of HER2-status for breast cancer patients is therefore crucial, which can be done by $I \mathrm{HC}, \mathrm{FISH}$, chromogenic in situ hybridisation (CISH), silver in situ hybridisation (SISH) and MLPA, as described by Moelans et al. ${ }^{34} \mathrm{IHC}$ is the most widely used since it is cheap, fast and simple. Staining is usually scored according to the DAKO guidelines as 0 (no staining or $<10 \%$ of the tumour cells are weak and incomplete, not circumferential), $1+(\geq 10 \%$ of the tumour cells is weak and incompletely stained), $2+(\geq 10 \%$ of the tumour cells are stained moderately intense [complete, or incomplete], or $<10 \%$ complete and intense), or $3+\left(>10 \%\right.$ is stained strongly and circumferentially). ${ }^{35}$ However, membrane staining can be influenced by fixation conditions, and $\mathrm{IHC}$ may miss amplified cases. ${ }^{36} \mathrm{FISH}$ is an effective and sensitive method to assess HER2 gene amplification status. However, it not a very practical primary screening tool for HER2 copy number status since it is time consuming, signals fades over time, tumour morphology is more difficult to appreciate and tumour heterogeneity can easily be missed. FISH is therefore usually performed as a second-line test for the $\mathrm{HC}(2+)$ equivocal group. The morphology with $\mathrm{CISH}$ and $\mathrm{SISH}$ is much better preserved, slides can be kept permanently, and it is easier to score. A recent review showed that HER2 IHC, FISH, CISH and SISH have good concordance between them. ${ }^{34}$

MLPA is a multiplex PCR technique that has been well validated for assessment of HER2 copy number status that works on only small amounts of fragmented DNA isolated from FFPE material (50-100 ng). MLPA can analyse up to 45 genes in one assay, and is thus able to simultaneously test different genes that are important for therapy selection or prognosis. Different studies have shown a good correlation between MLPA and IHC, FISH and CISH, ${ }^{36}$ but MLPA has not been directly validated with regard to its predictive value for trastuzumab response.

All these techniques show an overall good correlation with each other in comparative studies, but not without discrepancies both ways. ${ }^{34}$ As a result, there is no gold standard HER2 test, and no consensus on which of the above-mentioned techniques is to be preferred. A clinical diagnostic test for HER2 should accurately determine HER2 protein levels or gene copy number and should be reproducible and precise across multiple sites and users. Decisions on how to test can therefore be 
taken locally after proper local validation. Local preferences may also determine whether to use amplification tests only as reflex test in cases that are equivocal by $\mathrm{IHC}(2+)$, or as primary screening tests, or parallel next to $\mathrm{IHC}$.

Traditionally, polysomy of chromosome 17, where HER2 is located, has received much focus as a source of "pseudo-amplification" of HER2. Therefore, many FISH and CISH kits have dual probes for HER2 and a centromere 17 sequence, usually CEP17, to enable calculation of a HER2/CEP17 ratio, which according to guidelines, needs to be above $\geq 2.0$ to indicate "true" HER2 amplification. ${ }^{37}$ However, several studies have shown that polysomy of chromosome 17 is virtually absent in breast cancer, ${ }^{38}$ but that $17 \mathrm{q}$ rather shows a very complex gains and losses pattern, unrelated to the copy number status of the centromere. Correction with CEP17 probes may thereby provide misleading HER2 gene status assessment results, and the absolute copy number of HER2 likely serves best to indicate eligibility for trastuzumab therapy. ${ }^{39}$

\section{HER2 mutations}

In breast cancer, the potential therapeutic opportunities offered by rare (1\%-3\%) HER2 somatic mutations were long neglected, given the high prevalence of HER2 gene amplification. ${ }^{40}$ The development of massive parallel sequencing has shed new light on these activating mutations, mostly present in the tyrosine kinase domain (68\%, exons 19-20) and the extracellular domain $(20 \%$, exon 8$){ }^{41}$ Tumours considered to be HER2negative based on current guidelines may still be "addicted" to HER2 signalling due to HER2 activating mutations and hence may also benefit from HER2 targeting agents. Bose et al. have functionally characterised 13 HER2 mutations using in vitro kinase assays, protein structure analysis, cell culture and xenograft experiments. ${ }^{41}$ Seven of these mutations were activating mutations and all of these mutations were sensitive to the irreversible HER2/EGFR tyrosine kinase inhibitor neratinib, thus validating HER2 somatic mutations as drug targets for breast cancer treatment. ${ }^{41}$ This finding may very well lead to a new era for breast cancer management, where HER2 status is no longer solely determined by $\mathrm{IHC}$ and ISH techniques. However, clinical trials are first needed to test whether HER2mutated tumours are responsive to HER2-targeted drugs, and if so, which mutations are predictive of sensitivity to which agent.

\section{Gene expression arrays for HER2}

HER2 MRNA expression has also been explored as an alternative predictive test. There was a very good correlation between HER2 mRNA expression by microarray analysis and HER2 $\mathrm{IHC}^{21}$ There is proven predictive superiority of gene expression in discrepant cases. The "Targetprint" test was recently taken off the market. Oncotype Dx also contains the HER2 gene.

\section{PIK3CA mutations}

PIK3CA is an oncogene exhibiting oncogenic gain-of-function mutations in several cancers occurring in $20-40 \%$ (25\% by COSMIC; July 2013) of breast cancers. Several studies have suggested that PI3K pathway activation can negatively influence response to trastuzumab therapy, ${ }^{22,43}$ and endocrine therapy. ${ }^{44}$

Given their frequency, oncogenic capabilities, and the potential to induce resistance to commonly prescribed breast cancer treatments, the clinical relevance of PIK3CA mutations needs to be further clarified. In light of the emergence of a broadening array of anti-HER2 agents, determination of PIK3CA mutational status could have important clinical utility. In addition, inhibitors of the PI3K pathway that may reverse acquired and de novo drug resistance are currently in clinical development.

\section{BRCAness}

BRCAness refers to a tumour genotype as seen in BRCA germline related cancers. Such a phenotype predicts response to high dose chemotherapy ${ }^{45}$ and poly ADP ribose polymerase (PARP) inhibitors. Although yet far from completely defined, several techniques may be employed to identify BRCAness. Copy number profiles by array $\mathrm{CGH}$ or $\mathrm{MLPA}^{46}$ that point to BRCA germline mutation related cancers as described above may be useful. Further, BRCA1/2 promoter methylation may lead to a similar phenotype by inactivating BRCA1/2 function. ${ }^{47}$ Finally, tumours may be sequenced for sporadic BRCA1/2 mutations. This is expected to become more and more clinically important in breast cancer treatment.

\section{Testing of metastases}

Despite advances in treatment and screening modalities, approximately $30 \%$ of breast cancer patients eventually develop recurrent or metastatic disease. Although metastatic breast cancer remains essentially incurable and therefore the main cause of breast cancer related death, it is a highly understudied field. Growing evidence describes extensive differences in ER, PR and HER2 protein expression between primary breast tumours and their paired metastases (termed conversion).$^{48}$ Also, gene expression tests of these markers are worth executing, since the robustness of these tests can lead to a specific insight of the true extent of conversion. This can have an impact on systemic treatment choice and efficacy.

As mentioned earlier, many predictive and prognostic tests are already in use for primary tumours. Since metastases are a reflection of poor prognostic disease, especially predictive tests could be valuable to also test in this group. However, if described gene expression tests as Endopredict, MammaPrint and Oncotype Dx are equally able to foretell response to therapy in metastases, remains to be elucidated.

Next generation sequencing (NGS) of 'cancer-associated' genes already revealed high genomic concordance between breast tumours and paired metastases [data unpublished, submitted for publication], but new mutational events emerge during progression. Although not present in every patient, this clonal evolution can lead to new targetable aberrations in metastases relative to their tumour of origin. ${ }^{49}$ Currently, the above described potentially targetable ESR1 ligand-binding domain mutations occur especially in metastases and testing for this is probably the most established molecular test on breast cancer metastases.

Some of the differences between primary breast tumours and metastases can be explained by evolutionary pressure of systemic therapy. An example can be found in the PI3K/AKT/MTOR pathway, since adjuvant endocrine therapy can significantly increase p-mTOR, p-4EBP1 and p-p70S6K expression in metastases, indicating the compensatory activation of this pathway. ${ }^{50}$ Since this finding could forebode acquired endocrine therapy resistance, ${ }^{51}$ testing of metastases could prevent maladjusted treatment.

Recapitulating, molecular diagnostics could disclose new, (potentially) druggable targets in metastases compared to the primary tumour and could be extremely valuable for personalised cancer treatment in metastatic breast cancer.

\section{The role of next generation sequencing}

NGS allows for the simultaneous mutation analysis of either the genome, exome, or genes of interest. For routine diagnostics targeted NGS is a reliable assay to detect (hotspot) mutations in FFPE 
Table 2: Overview of various molecular tests for breast cancer and their current clinical utility

\begin{tabular}{|c|c|c|c|}
\hline Molecular alteration & Preferred test & Type of material & Current clinical utility \\
\hline \multicolumn{4}{|l|}{ Diagnostic tests } \\
\hline $\begin{array}{l}\text { BRCA1/2 promotor } \\
\text { hypermethylation }\end{array}$ & MLPA & Primary tumour & Hypermethylation denies an underlying BRCA1/2 germline mutation \\
\hline Copy number profiling & CGH, MLPA & Primary tumour & $\begin{array}{l}\text { Specific copy number profiles exist pointing to BRCA1/2 germline } \\
\text { mutations }\end{array}$ \\
\hline MYB-NFIB translocation & FISH, RT-PCR & Primary tumour & Points towards adenoid cystic carcinoma \\
\hline MYBL1-NFIB translocation & FISH, RT-PCR & Primary tumour & Points towards adenoid cystic carcinoma \\
\hline MYBL1-RAD51B translocation & FISH, RT-PCR & Primary tumour & Points towards adenoid cystic carcinoma \\
\hline ETV6-NTRK3 translocation & FISH, RT-PCR & Primary tumour & Points towards secretory carcinoma \\
\hline \multicolumn{4}{|l|}{ Prognostic tests } \\
\hline Endopredict & RT-PCR & Primary tumour & Predicts prognosis in ER+/HER2- N0-1 patients \\
\hline Mammaprint & Expression microarray & Primary tumour & Predicts prognosis in stage I-II patients with tumour size $<5 \mathrm{~cm}$ \\
\hline \multicolumn{4}{|l|}{ Predictive tests } \\
\hline Endopredict & RT-PCR & Primary tumour & $\begin{array}{l}\text { Personalising indications for chemotherapy and extended hormonal } \\
\text { treatment in ER+/HER2- N0-1 patients }\end{array}$ \\
\hline Mammaprint & Expression microarray & Primary tumour & $\begin{array}{l}\text { Omitting chemotherapy in hormonally treated clinically high/ } \\
\text { Mammaprint low risk stage I-II patients with tumour size }<5 \mathrm{~cm}\end{array}$ \\
\hline Oncotype Dx & RT-PCR & Primary tumour & $\begin{array}{l}\text { Deciding on chemotherapy in ER+ NO patients prognostically } \\
\text { equivocal based on standard features }\end{array}$ \\
\hline \multirow[t]{2}{*}{ ESR1 mutations } & Sequencing & Primary tumour & Point to resistance to ER inhibitors \\
\hline & & Blood & $\begin{array}{l}\text { Monitoring success of systemic treatment in case of ER mutation } \\
\text { positive metastatic patients }\end{array}$ \\
\hline ESR1 amplification & MLPA, FISH, qPCR, CGH & Primary tumour & Points to good prognosis and good response to tamoxifen \\
\hline HER2 amplification & MLPA, FISH, SISH, CISH & Primary tumour, distant metastases & Points to poor prognosis, good response to HER2 inhibitors \\
\hline PIK3CA mutations & Sequencing & Primary tumour, distant metastases & Point to poor response to trastuzumab and endocrine therapy \\
\hline BRCAness & Primary tumour & $\begin{array}{l}\text { CGH, MLPA, sequencing, } \\
\text { methylation analysis }\end{array}$ & $\begin{array}{l}\text { Points to good response to high dose chemotherapy and PARP } \\
\text { inhibitors }\end{array}$ \\
\hline
\end{tabular}

$\mathrm{CGH}=$ comparative genomic hybridisation; $E R$ = oestrogen receptor; FISH = fluorescent in situ hybridisation; HER2 = human epidermal growth factor receptor 2;

$M L P A=$ multiplex ligation-dependent probe amplification; $P A R P=$ poly ADP ribose polymerase; $R T-P C R=$ reverse transcription polymerase chain reaction.

samples. ${ }^{52}$ Although this technique is already extensively used for other tumour types (e.g., lung and colon tumours), mutation analysis of breast cancer is not yet routine practice. Implementation of NGS for breast cancer specifically analysing mutations in HER2, ER and PIK3CA, would provide information on treatment options. Potentially, BRCA mutation analysis can be added in the near future, if clinical studies show that the presence of a BRCA mutation is indication for treatment with PARP inhibitors. ${ }^{53}$

Next to mutation analysis, NGS allows for simultaneous copy number variation (CNV) detection, ${ }^{54}$ So HER2 amplification analysis can be performed with the same NGS assay as mutation analysis. For reliable CNV detection the percentage of neoplastic cells in the sample needs to be preferably at least $30 \%$. Since NGS provides a combined result of all normal and neoplastic cells within the tissue, the tumor information is diluted which might result in a negative CNV result when too few neoplastic cells are present in the sample.

NGS can in principle also be used for translocation and methylation detection in FFPE tissue. Currently, mostly genome wide RNA-seq and bisulfite sequencing approaches are used, which are not suitable for fast routine diagnostics. However, amplicon based NGS panels can enrich cDNA samples for fusions. Also, novel fusions, where only one of both fusion partners is known, can be detected using this technique. For methylation analysis, currently relatively large amounts of DNA are required due to the bisulfite treatment which degrades DNA, so this is not a technique which can easily be implemented in routine diagnostics. Also for methylation detection, a targeted NGS approach can be used, where the primers should be designed for bisulfite treated DNA. As both translocation and methylation analysis require a different starting material compared to mutation and CNV analysis (RNA and bisulfite treated DNA compared to untreated DNA), combining all techniques in one assay will only be possible for the sequencing step and not the preceding library preparation. In summary, NGS can currently be used to detect mutations and CNV in FFPE samples using a targeted approach.

\section{Liquid biopsy}

Although the above mentioned techniques can be performed reliably on tumour specimens and small biopsies, the question remains whether less invasive methods can be applied. Especially for the follow-up of patients to evaluate response to therapy, liquid biopsies seem to be an excellent alternative to tissue biopsies. Therefore, the tumour first needs to be screened for specific molecular alterations, which can subsequently be followed in the liquid biopsy, for example in blood samples. From the blood, either circulating tumour cells (CTCS) or circulating free tumour DNA (ctDNA) can be extracted to study these molecular alterations. 
Since the amount of CTCs or ctDNA in the blood is limited, very sensitive techniques are necessary, such as a digital PCR. This technique allows the detection of ctDNA mutations with a frequency of as low as $0.1 \%$. It is therefore also possible to detect minimal residual disease in blood samples. ${ }^{55}$ Alternatively, NGS can be used when a high coverage is used to allow very sensitive mutation detection. Rothé et al. described that known mutations can be detected in the blood using a targeted NGS approach when a coverage of $25,000 \mathrm{x}$ is used. ${ }^{56}$ Since the isolation of CTCs is more challenging than the isolation of ctDNA, clinical studies are ongoing to test whether CTCS can be used for monitoring treatment response..$^{56,57}$ In conclusion, liquid biopsies seem a reliable, non-invasive method to detect low-level molecular alterations for the follow-up of cancer treatment.

\section{Conclusions}

The portfolio of diagnostic molecular tests for breast cancer is constantly expanding. Tests that have been established for routine use include BRCA1/2 profile testing for detection of hereditary cancers by copy number MLPA/NGS and MS-MLPA, translocation testing for AdCC and secretory cancer, predictive gene expression profiling in clinically equivocal cases, HER2 amplification testing by ISH/MLPA, ER $\alpha$ mutation testing in metastases, HER2 and PIK3CA mutation testing in case of trastuzumab treatment, and BRCAness testing by MLPA (hypermethylation, copy number) or NGS (copy number, mutations). Table 2 provides an overview of the currently useful molecular methods in breast cancers, their preferred way of testing and the clinical utility.
1. Economopoulou P, Dimitriadis G, Psyrri A, Beyond BRCA: new hereditary breast cancer susceptibility genes, Cancer Treat Rev , 2015;41:1-8.

2. Vos S, Van der Groep P, Van der Wall E, Van Diest PJ, Hereditary Breast Cancer Syndromes: Molecular Pathogenesis and Diagnostics. eLS: New Jersey, US, John Wiley \& Sons, Ltd; 2001:1-16

3. Vos S, Moelans $\mathrm{CB}$, van Diest PJ, BRCA promoter methylation in sporadic versuS BRCA germline mutation-related breast cancers, Breast Cancer Res, 2017: in press.

4. Wessels LF van Welsem T Hart AA et al. Molecular

classification of breast carcinomas by comparative genomic hybridization: a specific somatic genetic profile for BRCA1 tumors, Cancer Res, 2002;62:7110-17.

5. Joosse SA, Brandwijk KI, Devilee P, et al., Prediction of BRCA2association in hereditary breast carcinomas using array-CGH Breast Cancer Res Treat, 2012;132:379-89.

6. Perou CM, Sorlie T, Eisen MB, et al., Molecular portraits of human breast tumours, Nature, 2000;406:747-52.

7. Sorlie T, Perou CM, Tibshirani R, et al., Gene expression patterns of breast carcinomas distinguish tumor subclasses with clinica implications, Proc Natl Acad Sci U S A, 2001;98:10869-74.

8. Ellis MJ, Suman VJ, Hoog J, et al., Randomized phase II neoadjuvant comparison between letrozole, anastrozole, and exemestane for postmenopausal women with estrogen receptor-rich stage 2 to 3 breast cancer: clinical and biomarker receptor-rich stage 2 to 3 breast cancer: clinical and biomarker
outcomes and predictive value of the baseline PAM50-based intrinsic subtype-ACOSOG Z1031, J Clin Oncol, 2011;29:2342-9.

9. Marchio C, Weigelt B, Reis-Filho JS, Adenoid cystic carcinomas of the breast and salivary glands (or 'The strange case of Dr Jekyll and Mr Hyde' of exocrine gland carcinomas), J Clin Pathol, 2010;63:220-8.

10. Brayer $\mathrm{KJ}$, Frerich $C A$, Kang $H$, Ness $S A$, Recurrent fusions in MYB and MYBL1 define a common, transcription factor-driven oncogenic pathway in salivary gland adenoid cystic carcinoma, Cancer DisCov, 2016;6:176-87.

11. Persson $M$, Andren $Y$, Mark J, et al., Recurrent fusion of MYB and NFIB transcription factor genes in carcinomas of the breast and head and neck, Proc Natl Acad SciU SA, 2009;106:18740-4.

12. Tognon C, Knezevich SR, Huntsman D, et al., Expression of the ETV6-NTRK3 gene fusion as a primary event in human secretory breast carcinoma, Cancer Cell, 2002:2:367-76.

13. Filipits $M$, Rudas $M$, Jakesz $R$, et al., A new molecular predictor of distant recurrence in ER-positive, HER2-negative breast cancer adds independent information to conventional clinical risk factors, Clin Cancer Res, 2011;17:6012-20

14. Sapino A, Roepman P, Linn SC, et al., MammaPrint molecular diagnostics on formalin-fixed, paraffin-embedded tissue, $J \mathrm{MOl}$ Diagn, 2014;16:190-7.

15. Paik $S$, Shak S, Tang $G$, et al., A multigene assay to predict recurrence of tamoxifen-treated, node-negative breast cancer, N Engl I Med, 2004;351:2817-26.

16. Gyanchandani R, Lin Y, Lin HM, et al., Intratumor Heterogeneity Affects Gene Expression Profile Test Prognostic Risk Stratification in Early Breast Cancer, Clin Cancer Res, 2016;22:5362-9.

17. Eden P, Ritz C, Rose C, et al., "Good Old" clinical markers have similar power in breast cancer prognosis as microarray gene expression profilers, Eur $」$ Cancer, 2004;40:1837-41.

18. Cuzick J, Dowsett M, Pineda S, et al., Prognostic value of a combined estrogen receptor, progesterone receptor, Ki-67, an human epidermal growth factor receptor 2 immunohistochemica score and comparison with the Genomic Health recurrence score in early breast cancer, I Clin Oncol, 2011;29:4273-8.

19. Dowsett M, Sestak I, Lopez-Knowles E, et al., Comparison of PAM50 risk of recurrence score with oncotype DX and IHC4 for predicting risk of distant recurrence after endocrine therapy J Clin Oncol, 2013;31:2783-90.

20. Dowsett M, Nielsen TO, A'Hern R, et al., Assessment of Ki67 in breast cancer: recommendations from the International Kib7 in Breast Cancer working group, J Natl Cancer Inst, 2011:103:1656-64.
21. Roepman P, Horlings HM, Krijgsman O, et al., Microarray-based determination of estrogen receptor, progesterone receptor, and HER2 receptor status in breast cancer, Clin Cancer Res, 2009;15:7003-11.

22. Robinson DR, WU YM, Vats $P$, et al., Activating ESR1 mutations in hormone-resistant metastatic breast cancer, Nat Genet, 2013;45:1446-51

23. Toy $\mathrm{W}$, Shen $\mathrm{Y}$, Won $\mathrm{H}$, et al., ESR1 ligand-binding domain mutations in hormone-resistant breast cancer, Nat Genet, 2013:45:1439-45.

24. Roodi N, Bailey LR, Kao WY, et al., Estrogen recepto gene analysis in estrogen receptor-positive and receptornegative primary breast cancer, I Natl Cancer Inst, 199515:87:446-51.

25. Jeselsohn R, Buchwalter G, De Angelis C, et al., ESR1 mutations-a mechanism for acquired endocrine resistance in breast cancer, Nat Rev Clin Oncol, 2015;12:573-83.

26. Merenbakh-Lamin K, Ben-Baruch N, Yeheskel A, et al., D538G mutation in estrogen receptor-alpha: A novel mechanism for acquired endocrine resistance in breast cancer, Cancer Res, 2013;73:6856-64

27. Sefrioui D, Perdrix A, Sarafan-Vasseur N, et al., Short report: Monitoring ESR1 mutations by circulating tumor DNA in aromatase inhibitor resistant metastatic breast cancer, Int J Cancer, 2015:137:2513-9.

28. Schiavon G, Hrebien S, Garcia-Murillas I, et al., Analysis of ESR1 mutation in circulating tumor DNA demonstrates evolution during therapy for metastatic breast cancer, Sci Trans/ Med 2015;7:313ra182.

29. AnguS L, Beije N, Jager A, et al., ESR1 mutations: Moving towards guiding treatment decision-making in metastatic breast cancer patients, Cancer Treat Rev, 2017;52:33-40.

30. Babyshkina N, Vtorushin S, Zavyalova M, et al., The distribution pattern of ER alpha expression, ESR1 genetic variation and expression of growth factor receptors: association with breast cancer prognosis in Russian patients treated with adjuvant tamoxifen, Clin Exp Med, 2016; [Epub ahead of print].

31. Moelans $C B$, Holst $F$, Hellwinkel $O$, et al., ESR1 amplification in breast cancer by optimized RNase FISH: frequent but low-leve and heterogeneous, PLOS One, 2013;8:e84189.

32. Lapidus RG, Ferguson AT, Ottaviano YL, et al., Methylation of estrogen and progesterone receptor gene $5^{\prime} \mathrm{CPG}$ islands correlates with lack of estrogen and progesterone receptor gene expression in breast tumors, Clin Cancer Res, 1996:2:805-10.

33. Ferguson AT, Lapidus RG, Baylin SB, Davidson NE, Demethylation of the estrogen receptor gene in estrogen receptor-negative breast cancer cells can reactivate estrogen receptor gene expression, Cancer Res, 1995;55:2279-83.

34. Moelans CB, de Weger RA, Van der Wall E, van Diest PJ, Current technologies for HER2 testing in breast cancer, Crit Rev Oncol Hematol, 2011;80:380-92.

35. Lim TH, Lim AS, Thike AA, et al., Implications of the updated 2013 American Society of Clinical Oncology/College of American Pathologists guideline recommendations on human epidermal growth factor receptor 2 gene testing using immunohistochemistry and fluorescence in situ hybridization Immunohistochemistry and fluorescence in situ hybridiza

36. Moelans CB, de Weger RA, Ezendam C, van Diest PJ, HER-2/ neu amplification testing in breast cancer by Multiplex Ligationdependent Probe Amplification: influence of manual- and lase dependent Probe Amplification: influence

37. Singh K, Tantravahi U, Lomme MM, et al., Updated 2013 College of American Pathologists/American Society of Clinica Oncology (CAP/ASCO) guideline recommendations for human epidermal growth factor receptor 2 (HER2) fluorescent in situ hybridization (FISH) testing increase HER2 positive and HER2 equivocal breast cancer cases; retrospective study of HER2 FISH results of 836 invasive breast cancers, Breast Cancer Res Treat, 2016;157:405-11.

38. Moelans $\mathrm{CB}$, Reis-Filho IS, van Diest PJ, Implications of rarity of chromosome 17 polysomy in breast cancer, Lancet Oncol, 2011;12:1087-9.
39. Moelans $\mathrm{CB}$, de Weger RA, van Diest PJ, Absence of chromosome 17 polysomy in breast cancer: analysis by CEP17 chromogenic in situ hybridization and multiplex ligationdependent probe amplification, Breast Cancer Res Treat, 2010;120:1-7

40. Weigelt B, Reis-Filho IS, Activating mutations in HER2: neu opportunities and neu challenges, Cancer Discov, 2013;3:145-7.

41. Bose R, Kavuri SM, Searleman AC, et al., Activating HER2 mutations in HER2 gene amplification negative breast cancer Cancer Discov, 2013:3:224-37.

42. Dave B, Migliaccio I, Gutierrez MC, et al., Loss of phosphatase and tensin homolog or phosphoinositol-3 kinase activation and response to trastuzumab or lapatinib in human epiderm growth factor receptor 2-overexpressing locally advance breast cancers, I Clin Oncol, 2011;29:166-73.

43. Wang L, Zhang Q, Zhang J, et al., PI3K pathway activation results in low efficacy of both trastuzumab and lapatinib, BMC Cancer, 2011;11:248.

44. Ma CX, Crowder RJ, Ellis MJ, Importance of PI3-kinase pathway in response/resistance to aromatase inhibitors, Steroids, 2011;76:750-2.

45. Vollebergh MA, Lips EH, Nederlof PM, et al., An aCGH classifier derived from BRCA1-mutated breast cancer and benefit of high-dose platinum-based chemotherapy in HER2-negative high-dose platinum-based chemotherapy in HER2-negat

46. Lips EH, Laddach N, Savola SP, et al., Quantitative copy number analysis by Multiplex Ligation-dependent Probe Amplification (MLPA) of BRCA1-associated breast cancer regions identifies BRCAness, Breast Cancer Res, 2011;13:R107.

47. Ibragimova I, Cairns P, Assays for hypermethylation of the BRCA1 gene promoter in tumor cells to predict sensitivity to PARP-inhibitor therapy, Methods Mol Biol, 2011;780:277-91.

48. Hoefnagel LD, Moelans CB, Meijer SL, et al., Prognostic value of estrogen receptor alpha and progesterone receptor conversion in distant breast cancer metastases, Cancer. 2012;118:4929-35

49. Bertucci F, Finetti P, Guille A, et al., Comparative genomic analysis of primary tumors and metastases in breast cancer, Oncotarget, 2016;7:27208-19.

50. Beelen K, Hoefnagel LD, Opdam M, et al., PI3K/AKT/MTOR pathway activation in primary and corresponding metastatic breast tumors after adjuvant endocrine therapy, Int $I$ Cancer 2014:135:1257-63

51. Cizkova M, Dujaric ME, Lehmann-Che J, et al., Outcome impact of PIK3CA mutations in HER2-positive breast cancer patient treated with trastuzumab, Br $\lrcorner$ Cancer, 2013;108:1807-9.

52. de Leng WW, Gadellaa-van Hooijdonk CG, Barendregt-Smoute FA, et al., Targeted next generation sequencing as a reliable diagnostic assay for the detection of somatic mutations in tumours using minimal DNA amounts from formalin fixed paraffin embedded material, PLOS One, 2016;11:e0149405.

53. Schouten PC, Dackus GM, Marchetti S, et al., A phase I followed by a randomized phase II trial of two cycles carboplatinolaparib followed by olaparib monotherapy versus capecitabine in BRCA1- or BRCA2-mutated HER2-negative advanced breast cancer as first line treatment (REVIVAL): study protocol for a randomized controlled trial, Trials, 2016:17:293.

54. Hoogstraat M, Hinrichs JW, Besselink NJ, et al., Simultaneous detection of clinically relevant mutations and amplifications for routine cancer pathology, J Mol Diagn, 2015;17:10-18.

55. Olsson E, Winter C, George A, et al., Serial monitoring of circulating tumor DNA in patients with primary breast cance for detection of occult metastatic disease, EMBO Mol Med, 2015;7:1034-47

56. Rothe F, Laes JF, Lambrechts D, SmeetS D, Vincent D, Maetens $\mathrm{M}$, et al. Plasma circulating tumor DNA as an alternative to metastatic biopsies for mutational analysis in breast cancer. Ann Oncol, 2014 Oct;25(10):1959-1965.

57. Ignatiadis M, Rack B, Rothe F, Riethdorf S, Decraene C, Bonnefo $\mathrm{H}$, et al. Liquid biopsy-based clinical research in early breast cancer: The EORTC 90091-10093 Treat CTC trial. Eur J Cancer 2016 Aug:63:97-104. 